\title{
ORIGINAL ARTICLE \\ Urodynamic investigations in patients with spinal cord injury: should the ice water test follow or precede the standard filling cystometry?
}

\author{
M Kozomara ${ }^{1}$, CHS Bellucci ${ }^{1}$, B Seifert ${ }^{2}$, TM Kessler ${ }^{1}$ and U Mehnert ${ }^{1}$
}

Objectives: To evaluate whether the ice water test (IWT) should be performed before or after the standard urodynamic investigation (UDI).

Patients and Methods: Two cohorts of patients suffering from neurogenic lower urinary tract dysfunction (NLUTD) due to spinal cord injury (SCl) were matched by lesion level and age. The patients of cohort $\mathrm{A}(n=55$, retrospective cohort) underwent the IWT before and the patients of cohort B $(n=110$, prospective cohort) after standard UDI. The IWT effect on urodynamic parameters has been compared between the two groups using the Mann-Whitney U-test for independent samples. UDI was performed according to good urodynamic practices recommended by the International Continence Society.

Results: The mean age of both cohorts was 49 years. Performing the IWT before versus after standard UDI resulted in a significantly lower maximum cystometric bladder capacity $(P=0.01)$, lower incidence of detrusor overactivity $(P=0.017)$ and lower maximum detrusor pressure during IWT $(P=0.04)$. All other urodynamic parameters assessed demonstrated no significant difference $(P>0.05)$. Conclusions: Our results are in line with findings from animal studies demonstrating a bladder cooling-induced gating effect on the micturition reflex volume threshold on the level of sacral interneurons. Since the IWT is an unphysiological investigation that might significantly bias subsequent urodynamics, we suggest that the IWT should not precede more physiological standard UDI.

Spinal Cord (2015) 53, 800-802; doi:10.1038/sc.2015.152; published online 22 September 2015

\section{INTRODUCTION}

The bladder cooling reflex is a spinal reflex that can be urodynamically investigated using the ice water test (IWT), that is, rapid infusion of cold saline into the bladder, which was first described 1957 by Bors and Blinn ${ }^{1}$ to differentiate between a lesion of the upper (positive bladder cooling reflex) or lower (negative bladder cooling reflex) motoneuron in spinal cord injury (SCI) patients. Studies in cats indicated that the bladder cooling reflex is a C-fiber-mediated lower motoneuron segmental reflex and that unmyelinated C-fibers are associated with specific cold receptors of the transient receptor potential family. ${ }^{2-4}$ Clinical investigations and immunohistochemical studies provided evidence that the mechanism of the bladder cooling reflex might be also true for humans. ${ }^{5}$

Since C-fibers seem to have a role in the pathomechanism of lower urinary tract symptoms (LUTS) not only in neurological patients, ${ }^{6-9}$ the clinical relevance of the IWT might even reach beyond the simple differentiation between an upper and lower motoneuron lesion.

Geirsson et al. ${ }^{10}$ elaborated on the use of the IWT in humans and suggested to infuse $100 \mathrm{ml}$ saline $<10^{\circ} \mathrm{C}$ at a speed of 100 to 300 $\mathrm{ml} \mathrm{s}^{-1}$ as standard for optimized bladder cooling reflex assessment and proposed a detrusor contraction in response to bladder cooling of $>30 \mathrm{~cm} \mathrm{H}_{2} \mathrm{O}$ as positive IWT result.

However, in daily urodynamic practice, it is still unclear, if the IWT should be performed before or after the standard urodynamic investigation (UDI) and in how far both investigations affect each other. Thus, we aimed to investigate the outcome of the IWT and its effect on urodynamic parameters when performed before or after standard UDI.

\section{PATIENTS AND METHODS}

This study was approved by the local ethics committee (Kantonale Ethikkommission Zürich) and conducted at the Spinal Cord Injury Centre, Balgrist University Hospital, Zürich, Switzerland.

The study comprised two independent cohorts: cohort A (retrospective data, IWT before UDI): urodynamic data from SCI patients with neurogenic lower urinary tract dysfunction (NLUTD) investigated at our center from January 2001 until September 2010 were reviewed. During this time period the IWT was exclusively performed prior to the standard UDI.

Cohort B (prospective data, IWT after UDI): from October 2010 to June 2012, SCI patients with NLUTD undergoing standard UDI and IWT were eligible for study participation. To be included, patients had to provide written informed consent. In this study part, the IWT was exclusively performed after standard UDI.

Exclusion criteria for both cohorts were current urinary tract infection, pregnancy, intradetrusor onabotulinumtoxin A injections within the past year before UDI and patient age $<18$ years old.

In cohort B, 242 patients could be prospectively enrolled and were matched by lesion level and age with 57 patients of cohort A. Propensity score matching resulted in 110 patients in cohort B and 55 patients in cohort A.

${ }^{1}$ Neuro-Urology, Spinal Cord Injury Center \& Research, University of Zürich, Balgrist University Hospital, Zürich, Switzerland and ${ }^{2}$ Epidemiology, Biostatistics and Prevention Institute, University of Zürich, Zürich, Switzerland

Correspondence: Dr U Mehnert, Neuro-Urology, Spinal Cord Injury Center \& Research, University of Zürich, Balgrist University Hospital, Forchstrasse 340, Zürich 8008, Switzerland.

E-mail: ulrich.mehnert@paralab.balgrist.ch

Received 9 March 2015; revised 20 July 2015; accepted 29 July 2015; published online 22 September 2015 
All methods, definitions, terms and units used in this study are in accordance with the standards recommended by the International Continence Society. ${ }^{11}$

\section{Urodynamic investigation}

UDIs were done in accordance to good urodynamic practices recommended by the International Continence Society. ${ }^{12}$ A multichannel urodynamic system (Sedia, Givisiez, Switzerland) was applied for all measurements. Pelvic floor electromyographical data were recorded via two perineal surface electrodes. Filling sensations were documented when applicable. Standard UDI was performed using body warm saline and a filling speed of $20-30 \mathrm{ml} \mathrm{min}^{-1}$. The IWT was conducted with $4^{\circ} \mathrm{C}$ saline and a filling speed of $100 \mathrm{ml} \mathrm{min}^{-1}$.

\section{Outcome measures}

From storage phase of standard UDI: maximum cystometric bladder capacity $(\mathrm{ml})$, bladder volume at first detrusor overactivity $(\mathrm{ml})$, incidence of detrusor overactivity (\%), maximum detrusor pressure $\left(\mathrm{cm} \mathrm{H}_{2} \mathrm{O}\right)$ and bladder compliance during $\left(\Delta \mathrm{ml} \Delta \mathrm{cm}^{-1} \mathrm{H}_{2} \mathrm{O}\right)$.

From IWT: maximum detrusor pressure $\left(\mathrm{cm} \mathrm{H}_{2} \mathrm{O}\right)$.

\section{Statistical analyses}

Data were approximately normally distributed and are shown as mean \pm s.d. To limit the observational character of the study we analyzed a propensity matched sample. For the computation of the propensity score, age and lesion level were included into a logistic regression with the IWT performed before or after the standard UDI as dependent variable. The validity of logistic regression was assessed using the Hosmer-Lemeshow test. Propensity matching was

Table 1 Baseline characteristics of the patients of the two cohorts

\begin{tabular}{|c|c|c|c|}
\hline & $\begin{array}{c}\text { Cohort A (IWT } \\
\text { before FC) } \\
\text { mean } \pm \text { s.d. } \\
\text { (range) }\end{array}$ & $\begin{array}{c}\text { Cohort B (IWT } \\
\text { after FC) } \\
\text { mean } \pm \text { s.d. } \\
\text { (range) }\end{array}$ & P-value \\
\hline $\begin{array}{l}\text { No. of patients (all with NLUTD } \\
\text { due to } \mathrm{SCI} \text { ) }\end{array}$ & 55 & 110 & \\
\hline Age in years, mean \pm s.d. (range) & $49 \pm 15(20-85)$ & $49 \pm 15(20-81)$ & 0.966 \\
\hline \multicolumn{4}{|l|}{ Gender distribution } \\
\hline Female, $n(\%)$ & $25(45)$ & $19(17)$ & $<0.001$ \\
\hline Male, $n(\%)$ & $30(55)$ & $91(83.8)$ & \\
\hline \multicolumn{4}{|l|}{ Level of lesion } \\
\hline Cervical, $n(\%)$ & $17(33)$ & $38(34)$ & 0.906 \\
\hline Thoracic, $n(\%)$ & $35(67)$ & $75(66)$ & \\
\hline
\end{tabular}

Abbreviations: FC, filling cystometry; IWT, ice water test; NLUTD, neurogenic lower urinary tract dysfunction; $\mathrm{SCl}$, spinal cord injury.

Matching of subjects between cohorts A and B was performed using propensity score analysis with a proportion of $1: 2$ performed using the package Matching in $\mathrm{R} .{ }^{13}$ Cohorts A and B were matched with a proportion of 1:2. Patients of cohorts $\mathrm{A}$ and $\mathrm{B}$ were compared using the Mann-Whitney $U$-test. Statistical analyses were performed applying IBM SPSS version 22 (IBM, Armonk, NY, USA). A $P$-value $<0.05$ was considered statistically significant.

\section{RESULTS}

The patients' characteristics of cohorts A and B are shown in Table 1.

Table 2 summarizes the outcome parameters of both cohorts. Performing the IWT before versus after standard UDI resulted in a significantly lower maximum detrusor pressure during IWT $(P=0.04)$ and a lower maximum cystometric bladder capacity $(P=0.01)$, lower incidence of detrusor overactivity $(P=0.017)$ during subsequent standard UDI and lower maximum detrusor pressure during IWT $(P=0.04)$. All other urodynamic parameters assessed demonstrated no significant difference $(P>0.05)$.

\section{DISCUSSION}

Our current findings suggest that performing the IWT before the standard UDI has effects on the outcome of the UDI, namely a decrease in maximum cystometric bladder capacity and bladder volume at first detrusor overactivity. Jiang et al. assumed based on findings from animal studies with cats that the dynamic cooling reflex is a C-fiber-mediated reaction evoked by rapid cooling of the bladder, which leads to a reflex discharge in bladder preganglionic neurons. ${ }^{14,15}$ Thereafter the cold evoked efferent activity persists for several tens of seconds and leads to a reduction of the threshold volume of the A-delta mechanoreceptor driven micturition reflex. ${ }^{14,15}$ In line with the observations of Jiang et al., ${ }^{15}$ we found a reduced volume threshold for detrusor overactivity but no changes in compliance or maximum detrusor pressure, indicating that the A-delta mechanoreceptor driven micturition reflex itself remains unchanged but is gated at a lower threshold volume.

Jiang et al. suggested that this gating of the micturition reflex occurs rather on a spinal level through preganglionic interneurons than via the pontine micturition center (PMC) because of the observation that bladder C- and A-delta fiber-mediated reflexes are modulated in parallel by a spinal enkephalinergic recurrent inhibitory mechanism. ${ }^{15}$ This recurrent inhibition, which has been demonstrated to originate from axon collaterals of bladder preganglionic neurons, ${ }^{16,17}$ is supposed to act at spinal interneurons prior to the preganglionic lower urinary tract (LUT) neurons. ${ }^{15,18}$

The PMC in contrast induces the micturition reflex via one long descending pathway to the sacral spinal cord where the reflex is further mediated by segmental interneurons as an all-or-none response. ${ }^{19}$ Only the latter reflex enhanced component but not the descending component from the PMC has been demonstrated to be susceptible to

Table 2 Outcome parameters comparing IWT performed before and after standard urodynamic

\begin{tabular}{|c|c|c|c|}
\hline & Cohort A (IWT before FC) mean \pm s.d. (range) & Cohort B (IWT after FC) mean \pm s.d. (range) & P-value \\
\hline Maximum cystometric bladder capacity (ml) & $444 \pm 158(40-800)$ & $611 \pm 276(110-1400)$ & 0.01 \\
\hline Bladder volume at first detrusor overactivity $(\mathrm{ml})$ & $288 \pm 133(25-600)$ & $360 \pm 190(35-1060)$ & 0.059 \\
\hline Incidence of detrusor overactivity & $71 \%(n=39)$ & $86 \%(n=95)$ & 0.017 \\
\hline $\begin{array}{l}\text { Maximum detrusor pressure during storage phase of standard UDI } \\
\left(\mathrm{cm} \mathrm{H}_{2} \mathrm{O}\right)\end{array}$ & $32 \pm 15(10-67)$ & $39 \pm 20(11-99)$ & 0.102 \\
\hline Maximum detrusor pressure during IWT $\left(\mathrm{cm} \mathrm{H}_{2} \mathrm{O}\right)$ & $36 \pm 31(2-127)$ & $43 \pm 26(0-116)$ & 0.04 \\
\hline Bladder compliance $\left(\Delta \mathrm{ml} / \Delta \mathrm{cm} \mathrm{H} \mathrm{H}_{2} \mathrm{O}\right)$ & $46 \pm 26(13-150)$ & $56 \pm 51(9-350)$ & 0.597 \\
\hline
\end{tabular}

Abbreviation: IWT, ice water test. 
modulation by recurrent inhibition. ${ }^{15}$ This would also explain why the bladder cooling-induced modulation of the micturition reflex can be also observed in our SCI study population.

van Meel et al. ${ }^{20}$ observed similar effects during repeated IWT with an increase of positive IWT results with each repetition. Although repeated IWT in conjunction with LUT current perception threshold measurement can be helpful to identify unsuspected neurological pathologies in patients with assumed idiopathic LUTS, ${ }^{20}$ the IWT still remains an unphysiological stimulation that can significantly bias subsequent investigations such as standard UDI. Since the standard UDI aims to reflect the daily LUT function as close and physiological as possible, we recommend-based on our findings - to perform the IWT after the standard UDI to obtain unbiased and consequently comparable UDI results. If in patients with NLUTD repeated standard UDIs as recommended previously ${ }^{21}$ do not reveal any findings correlating with the daily symptoms, an IWT might be used as provocation test to reveal masked pathologies such as detrusor overactivity but after the standard UDI.

Considering that the human bladder seems to be innervated by A-delta and C-fibers only, ${ }^{22}$ urodynamic testing of both fiber types offers the possibility to more comprehensively assess the underlying pathology of the clinical symptoms. This might be especially relevant, since cold sensitive transient receptor potential channels, that is, TRPM8, and cold sensitive C-fibers have been specifically associated with lower urinary tract dysfunction. ${ }^{7,22,23}$

Because of their high intravesical pressure thresholds, C-fibers are in contrast to Ad-fibers considered less relevant for normal, gradual development of bladder sensations, such as first sensation of filling, first desire to void and strong desire to void. ${ }^{22}$ However, C-fiber mechanosensitivity might change in response to chemical or thermal stimuli or alterations in neuronal control such as SCI. ${ }^{24}$ These nociceptive properties of $\mathrm{C}$-fibers might be related to the finding that they express various receptors including those of the transient receptor potential family. ${ }^{22}$ In conjunction with their abundant distribution within the LUT which accounts for approximately two thirds of bladder afferent neurons in rats, ${ }^{25} \mathrm{C}$-fibers represent a relevant sensory component of the LUT that, in case of aberrant functioning, might be accounted for several irritative symptoms of which some are clinically summarized as overactive bladder. ${ }^{26}$ Hence, assessment of such C-fibers in patients might improve our understanding on LUT symptoms. ${ }^{6,27}$

Although there is a lack of validated standardization on performing and interpreting the IWT, the description by Geirsson et al. ${ }^{10}$ and the review by Al-Hayek et al. ${ }^{28}$ provides a useful first recipe to perform the IWT. Since the IWT in our study has been performed in the same manner in all patients, a comparison between our cohorts is valid.

There are limitations of this study that should be considered: the study design was not fully prospective, rather one cohort consisted of retrospective data only. The presented results are based on findings in patients with NLUTD because of SCI. Thus, our findings cannot be generalized beyond this group.

In conclusion, this is the first study to evaluate the effect of the IWT on the standard UDI in SCI patients with NLUTD. Since the IWT is a rather unphysiological investigation that might significantly bias subsequent urodynamics, we suggest that the IWT should not precede more physiological standard UDI.

\section{DATA ARCHIVING}

There were no data to deposit.

\section{CONFLICT OF INTEREST}

The authors declare no conflict of interest.

\section{ACKNOWLEDGEMENTS}

This study was funded by the Swiss Continence Foundation (www.swisscontinencefoundation.ch) and the Clinical Research Priority Program NeuroRehab of the University of Zürich. The Swiss Continence Foundation and the Clinical Research Priority Program NeuroRehab of the University of Zürich had no role in study design, data collection and analysis, decision to publish or preparation of the manuscript.

1 Bors EH, Blinn KA. Spinal reflex activity from the vesical mucosa in paraplegic patients. AMA Arch Neurol Psychiatry 1957; 78: 339-354.

2 Raz S. Objective assessment of bladder response in ice water test. J Urol 1973; 109 603-604.

3 Fall M, Lindstrom S, Mazieres L. A bladder-to-bladder cooling reflex in the cat. J Physiol 1990; 427: 281-300.

4 Mazieres $\mathrm{L}$, Jiang $\mathrm{C}$, Lindstrom $\mathrm{S}$. The $\mathrm{C}$ fibre reflex of the cat urinary bladder. J Physiol 1998; 513: 531-541.

5 Geirsson G, Lindstrom S, Fall M. The bladder cooling reflex and the use of cooling as stimulus to the lower urinary tract. J Urol 1999; 162: 1890-1896.

6 Mukerji G, Waters J, Chessell IP, Bountra C, Agarwal SK, Anand P. Pain during ice water test distinguishes clinical bladder hypersensitivity from overactivity disorders. BMC Urol 2006; 6: 31 .

7 Mukerii G, Yiangou Y, Corcoran SL, Selmer IS, Smith GD, Benham CD et al. Cool and menthol receptor TRPM8 in human urinary bladder disorders and clinical correlations. BMC Urol 2006; $6: 6$.

8 Wyndaele JJ, De Wachter S. The sensory bladder (1): an update on the different sensations described in the lower urinary tract and the physiological mechanisms behind them. Neurourol Urodyn 2008; 27: 274-278.

9 Yoshimura N, Chancellor MB. Current and future pharmacological treatment for overactive bladder. J Urol 2002; 168: 1897-1913.

10 Geirsson G, Lindstrom S, Fall M. Pressure, volume and infusion speed criteria for the ice-water test. Br J Urol 1994; 73: 498-503.

11 Abrams P, Cardozo L, Fall M, Griffiths D, Rosier P, Ulmsten U et al. The standardisation of terminology of lower urinary tract function: report from the Standardisation Sub-committee of the International Continence Society. Neurourol Urodyn 2002; 21: 167-178.

12 Schafer W, Abrams P, Liao L, Mattiasson A, Pesce F, Spangberg A et al. Good urodynamic practices: uroflowmetry, filling cystometry, and pressure-flow studies. Neurourol Urodyn 2002; 21: 261-274.

13 Sekhon JS. Multivariate and Propensity Score Matching Software with Automated Balance Optimization: the Matching Package for R. J Stat Softw 2011; 42: 1-52.

14 Jiang $\mathrm{CH}$, Mazieres L, Lindstrom S. Cold- and menthol-sensitive C afferents of cat urinary bladder. J Physiol 2002; 543: 211-220.

15 Jiang $\mathrm{CH}$, Mazieres L, Lindstrom S. Gating of the micturition reflex by tonic activation of bladder cold receptors in the cat. Neurourol Urodyn 2009; 28: 555-560.

16 De Groat WC, Ryall RW. Recurrent inhibition in sacral parasympathetic pathways to the bladder. J Physiol 1968; 196: 579-591.

17 Morgan CW. Axons of sacral preganglionic neurons in the cat: II. Axon collaterals. Neurocytol 2001; 30: 767-787.

18 de Groat WC. Mechanisms underlying recurrent inhibition in the sacral parasympathetic outflow to the urinary bladder. J Physiol 1976; 257: 503-513.

19 Michels L, Blok BF, Gregorini F, Kurz M, Schurch B, Kessler TM et al. Supraspinal Control of Urine Storage and Micturition in Men-An fMRI Study. Cereb Cortex. e-pub ahead of print.

20 van Meel TD, de Wachter S, Wyndaele JJ. Repeated ice water tests and electrical perception threshold determination to detect a neurologic cause of detrusor overactivity. Urology 2007; 70: 772-776.

21 Bellucci CH, Wöllner J, Gregorini F, Birnböck D, Kozomara M, Mehnert U et al. Neurogenic lower urinary tract dysfunction-do we need same session repeat urodynamic investigations? J Urol 2012; 187: 1318-1323.

22 de Groat WC, Yoshimura N. Afferent nerve regulation of bladder function in health and disease. Handb Exp Pharmacol 2009; 194: 91-138.

23 de Groat WC, Yoshimura N. Changes in afferent activity after spinal cord injury. Neurourol Urodyn 2010; 29: 63-76.

24 Fowler CJ, Griffiths D, de Groat WC. The neural control of micturition. Nat Rev Neurosci 2008; 9: 453-466.

25 Yoshimura N, Erdman SL, Snider MW, de Groat WC. Effects of spinal cord injury on neurofilament immunoreactivity and capsaicin sensitivity in rat dorsal root ganglion neurons innervating the urinary bladder. Neuroscience 1998; 83: 633-643.

26 Juszczak K. Thor PJ. The basic neurophysiologic concept of lower urinary tract function-the role of vanilloid TRPV1 receptors of urinary bladder afferent nerve endings. Adv Clin Exp Med 2012; 21: 417-421.

27 Ismael SS, Epstein T, Bayle B, Denys P, Amarenco G. Bladder cooling reflex in patients with multiple sclerosis. J Urol 2000; 164: 1280-1284.

28 Al-Hayek S, Abrams P. The 50-year history of the ice water test in urology. J Urol 2010 183: $1686-1692$. 